\title{
A GENERALIZATION OF THE GLOBAL LIMIT THEOREMS OF R.P. AGNEW
}

\author{
ANDREW ROSALSKY \\ Department of Statistics \\ University of Florida \\ Gainesville, Florida 32611 U.S.A.
}

(Received May 21, 1987 and in revised form August 24, 1987)

ABSTRACT. For distribution functions $\left\{F_{n}, n \geq 0\right\}$, the relationship between the weak convergence of $F_{n}$ to $F_{0}$ and the convergence of $\int_{R} \phi\left(\left|F_{n}-F_{0}\right|\right) d x$ to 0 is studied where $\phi$ is a nonnegative, nondecreasing function. Sufficient and, separately, necessary conditions are given for the latter convergence thereby generalizing the so-called global limit theorems of Agnew wherein $\phi(t)=|t|^{r}$. The sufficiency results are shown to be sharp and, as a special case, yield a global version of the central limit theorem for independent random variables obeying the Liapounov condition. Moreover, weak convergence of distribution functions is characterized in terms of their almost everywhere limiting behavior with respect to Lebesgue measure on the line.

KEY WORDS AND PHRASES. Distribution function, global limit theorem, weak convergence, complete convergence, almost everywhere convergence, uniform convergence, sums of independent random variables, central limit theorem, Liapounov condition.

1980 AMS SUBJECT CLASSIFICATION CODES. Primary 60F05; Secondary 60B10, $60 \mathrm{E} 05$.

\section{INTRODUCTION.}

Let $\mathcal{F}$ denote the class of all nondecreasing, left continuous functions $F$ defined on $R=(-\infty, \infty)$ such that $\lim _{x \rightarrow-\infty} F(x) \geq 0$ and $\lim _{x \rightarrow \infty} F(x) \leq 1$. A distribution function is a member of $F$ satisfying $\lim _{x \rightarrow-\infty} F(x)=0$ and $\lim _{x \rightarrow \infty} F(x)=1$. For $F \in J$, let $C(F)$ denote the continuity set of $F$, that is, $C(F)=\{x \in R: F$ is continuous at $x\}$. A sequence of distribution functions $\left\{F_{n}, n \geq 1\right\}$ is said to converge weakly to a function $F_{0} \in \mathcal{F}$, denoted $\mathrm{F}_{\mathrm{n}} \stackrel{\mathrm{w}}{\rightarrow} \mathrm{F}_{0}$, if

$$
\lim _{n \rightarrow \infty} F_{n}(x)=F_{0}(x), \text { all } x \in C\left(F_{0}\right) \text {. }
$$

If $\left\{F_{n}, n \geq 1\right\}$ and $F_{0}$ are distribution functions with $F_{n} \stackrel{w}{\rightarrow} F_{0}$, then the sequence $\left\{F_{n}, n \geq 1\right\}$ is said to converge completely to $F_{0}$ and this is denoted by $F_{n} \stackrel{c}{\rightarrow} F_{0} \cdot$

For distribution functions $\left\{F_{n}, n \geq 0\right\}, R . P$. Agnew [1] was apparently the first to study the relationship between

$$
\lim _{n \rightarrow \infty} F_{n}(x)=F_{0}(x) \text {, all } x \in R
$$


and the convergence to 0 as $n \rightarrow \infty$ of the integrals $I_{n} \equiv \int_{R}\left|F_{n}(x)-F_{0}(x)\right|^{r} d x$ where $r>0$. A theorem whose conclusion is of the form

$$
\lim _{n \rightarrow \infty} \int_{R} \phi\left(\left|F_{n}(x)-F_{0}(x)\right|\right) d x=0
$$

where $\phi$ is a nonnegative, nondecreasing function is a so-called global limit theorem and it clearly supplements a limit theorem whose conclusion is (1.1) (or (1.2)) wherein convergence is pointwise in $x$ over $C\left(F_{0}\right)$ (or $R$ ). Agnew showed that the modes of convergence $(1.2)$ and $I_{n} \rightarrow 0$ are indeed rather closely related. Specifically, he proved the following two theorems.

THEOREM A (Agnew [1]). Let $\left\{F_{n}, n \geq 0\right\}$ be distribution functions such that

$$
\int_{R} x d F_{n}(x)=0, \quad \int_{R} x^{2} d F_{n}(x)=1, n \geq 0
$$

If $\lim _{n \rightarrow \infty} F_{n}(x)=F_{0}(x)$, all $x \in R$, then for all $r>1 / 2$,

$$
\lim _{n \rightarrow \infty} \int_{R}\left|F_{n}(x)-F_{0}(x)\right|^{r} d x=0
$$

The next theorem is a sort of converse to the preceding one. Note that condition (1.4) is not part of the hypotheses.

THEOREM B (Agnew [1]). Let $\left\{F_{n}, n \geq 0\right\}$ be distribution functions and suppose that $\mathrm{F}_{0}$ is continuous and strictly increasing over $\mathrm{R}$. If for some $r>0$

$$
\lim _{n \rightarrow \infty} \int_{R}\left|F_{n}(x)-F_{0}(x)\right|^{r} d x=0
$$

then

$$
\lim _{n \rightarrow \infty} \sup _{x \in R}\left|F_{n}(x)-F_{0}(x)\right|=0
$$

Throughout, the symbol dx signifies integration with respect to Lebesgue measure on $\mathrm{R}$ and the abbreviation a.e. stands for almost everywhere with respect to Lebesgue measure.

In this paper, we will generalize Theorems $A$ and $B$ by providing sufficient (Theorem 1) and, separately, necessary (Theorem 3) conditions for a relation of the form (1.3). New results concerning weak and uniform convergence of distribution functions are obtained in the process. The sharpness of Theorem 1 is established via an example.

Agnew [1] applied Theorem $A$ to the case where $\mathrm{F}_{0}$ is the $\mathrm{N}(0,1)$ distribution function and $F_{n}$ is the distribution function of $\Sigma_{j=1}^{n} x_{j} / \sqrt{n}, n \geq 1$, where $\left\{x_{n}, n \geq 1\right\}$ are independent, identically distributed (i.i.d.) random variables with mean 0 , variance 1 and obtained what he terms a global version of the central limit theorem (CLT). This will be discussed and extended in section 3.

Some global limit theorems were obtained by Kruglov [2] in the cases where (i) $F_{n}$ is the distribution function of the row sum of a double array of rowwise independent random variables and $(i i) F_{n}$ is the distribution function of a suitably normed and centered sum of i.i.d. random variables. Although in the current work 
none of those results will be applied or extended, the author takes great pleasure to acknowledge that it is Professor Kruglov's article which helped him formulate some of the results herein and taught him some of the general techniques which are employed to establish them.

\section{MAINSTREAM.}

Theorem 1 may now be established. It is a generalization of Theorem $A$ and this is perhaps most apparent from Corollary 2.

THEOREM 1. Let $\left\{F_{n}, n \geq 1\right\}$ be distribution functions and suppose that there exists a continuous function $g$ on $[0, \infty)$ satisfying

$$
0<\mathrm{g}(\mathrm{x}) \uparrow \text { as } 0<\mathrm{x} \uparrow
$$

and

$$
\sup _{n \geq 1} \int_{R} g(|x|) d F_{n}(x)<\infty .
$$

Let $\phi$ be a nondecreasing function on $[0, \infty)$ which is continuous at 0 with $\phi(0) \geq 0$, $\phi(x)>0$, some $x$, and such that

$$
\int_{[1, \infty)} \phi\left(\frac{C}{g(x)}\right) d x<\infty, \text { a } 11 c>0
$$

If $\mathrm{F}_{\mathrm{n}} \stackrel{\mathrm{w}}{\rightarrow} \mathrm{F}_{0}$ for some $\mathrm{F}_{0} \in \mathbf{3}$ then

$$
\mathrm{F}_{\mathrm{n}} \stackrel{\mathrm{c}}{\rightarrow} \mathrm{F}_{0}
$$

and

$$
\lim _{n \rightarrow \infty} \int_{R} \phi\left(\left|F_{n}(x)-F_{0}(x)\right|\right) d x=0
$$

PROOF. Note at the outset that (2.3) and the monotonicity of $\phi$ ensure that necessarily $\phi(0)=0$. It will be shown firstly that $g(x)+\infty$ as $x+\infty$. By hypothesis, $\phi\left(x_{0}\right)>0$ for some $x_{0}$. Now if $g(x) \uparrow B<\infty$, then

$$
\int_{[1, \infty)} \phi\left(\frac{\mathrm{Bx}_{0}}{\mathrm{g(x)}}\right) \mathrm{dx} \geq \int_{[1, \infty)} \phi\left(\mathrm{x}_{0}\right) \mathrm{dx}=\infty
$$

which contradicts $(2.3)$. Thus $g(x)+\infty$.

Next, note that for $n \geq 1$ and $a>0$

$$
\begin{aligned}
\int_{R} g(|x|) d F_{n}(x) & =\int_{[|x| \geq a]} g(|x|) d F_{n}(x)+\int_{[|x|<a]} g(|x|) d F_{n}(x) \\
& \geq g(a) \int_{[|x| \geq a]} 1 d F_{n}(x)
\end{aligned}
$$

implying via $(2.2)$ and $g(x) \uparrow_{\infty}$ that

$$
\lim _{a \rightarrow \infty} \sup _{n \geq 1} \int_{[|x| \geq a]} 1 d F_{n}(x) \leq \lim _{a \rightarrow \infty} \frac{\sup _{n \geq 1} \int_{R} g(|x|) d F_{n}(x)}{g(a)}=0,
$$

whence (see, e.g., Chow and Teicher [3, p. 253]) relation (2.4) obtains. 
It will now be shown that

$$
\int_{R} g(|x|) d F_{0}(x)<\infty
$$

For arbitrary $a$ and $b$ in $C\left(F_{0}\right)$ with $a<b$, it follows from the Helly-Bray lemma (see, e.g., Chow and Teicher [3, p. 251]) and the monotone convergence theorem that

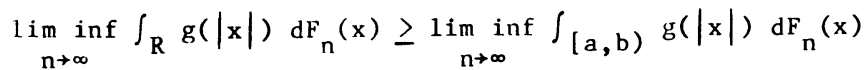

$$
\begin{aligned}
& =\int_{[a, b)} g(|x|) d F_{0}(x) \stackrel{\substack{a+\infty \\
b+\infty}}{\longrightarrow} \int_{R} g(|x|) d F_{0}(x)
\end{aligned}
$$

and so (2.6) follows recalling $(2.2)$.

Hence, in view of $(2.2)$ and $(2.6)$, a number $M<\infty$ may be chosen so that

$$
\int_{R} g(|x|) d F_{n}(x) \leq M, \quad n \geq 0
$$

Let $\left\{x_{n}, n \geq 0\right\}$ be random variables on some probability space $(\Omega, \alpha, P)$ such that the distribution function of $x_{n}$ is $F_{n}, n \geq 0$. Using the monotonicity of $\phi$, the Markov inequality, and (2.7), it follows that for $n \geq 1$ and $x \leq-1$

$$
\begin{aligned}
& \phi\left(\left|F_{n}(x)-F_{0}(x)\right|\right) \leq \phi\left(P\left\{x_{n}<x\right\}+P\left\{x_{0}<x\right\}\right) \\
\leq & \phi\left(P\left\{g\left(\left|x_{n}\right|\right) \geq g(|x|)\right\}+P\left\{g\left(\left|x_{0}\right|\right) \geq g(|x|)\right\}\right) \\
\leq & \phi\left(\frac{E g\left(\left|x_{n}\right|\right)+E g\left(\left|x_{0}\right|\right)}{g(|x|)}\right) \leq \phi\left(\frac{2 N}{g(|x|)}\right)
\end{aligned}
$$

and that for $\mathrm{n} \geq 1$ and $\mathrm{x} \geq 1$

$$
\begin{aligned}
& \phi\left(\left|F_{n}(x)-F_{0}(x)\right|\right)=\phi\left(\left|1-P\left\{X_{n} \geq x\right\}-\left(1-P\left\{X_{0} \geq x\right\}\right)\right|\right) \\
\leq & \phi\left(P\left\{g\left(\left|x_{n}\right|\right) \geq g(x)\right\}+P\left\{g\left(\left|x_{0}\right|\right) \geq g(x)\right\}\right) \\
\leq & \phi\left(\frac{E g\left(\left|x_{n}\right|\right)+E g\left(\left|x_{0}\right|\right)}{g(x)}\right) \leq \phi\left(\frac{2 M}{g(x)}\right) .
\end{aligned}
$$

Hence for $n \geq 1$,

$$
\phi\left(\left|F_{n}(x)-F_{0}(x)\right|\right) \leq \begin{cases}\phi(1), & |x|<1 \\ \phi\left(\frac{2 M}{g(|x|)}\right), & |x| \geq 1\end{cases}
$$

which is Lebesgue integrable over $R$ by (2.3). Now $\left|F_{n}-F_{0}\right| \rightarrow 0$ a.e. in view of (2.4) (see Theorem 2). Then since $\phi$ is continuous at 0 and $\phi(0)=0, \phi\left(\left|F_{n}-F\right|\right)+0$ a.e. and so (2.5) then follows via the Lebesgue dominated convergence theorem.

The following corollary follows immediately from Theorem 1 .

COROLLARY 1. Let $\left\{F_{n}, n \geq 1\right\}$ be distribution functions and suppose that there exists a continuous function $g$ on $[0, \infty)$ satisfying $(2.1),(2.2)$, and

$$
\int[1, \infty) \frac{1}{(g(x))^{r}} d x<\infty \text {, some } r>0 \text {. }
$$


If $\mathrm{F}_{\mathrm{n}} \stackrel{\mathrm{w}}{\rightarrow} \mathrm{E}_{0}$ for some $\mathrm{F}_{0} \in \mathcal{F}$, then $(2.4)$ obtains and

$$
\lim _{n \rightarrow \infty} \int_{R}\left|F_{n}(x)-F_{0}(x)\right|^{r} d x=0
$$

The next corollary was obtained by Nishimura [4] in the case $p>1, r=1$, $F_{n}(0)=0, n \geq 0$. However, Nishimura's argument is incomplete in that it was not shown that

$$
\int_{[0, \infty)} x^{p} d F_{0}(x)<\infty
$$

COROLLARY 2. Let $\left\{F_{n}, n \geq 1\right\}$ be distribution functions and suppose that

$$
\sup _{n \geq 1} \int_{R}|x|^{p} d_{n}(x)<\infty, \text { some } p>0 \text {. }
$$

If $\mathrm{F}_{\mathrm{n}} \stackrel{\mathrm{w}}{\rightarrow} \mathrm{F}_{0}$ for some $\mathrm{F}_{0} \in \mathbb{J}$, then $(2.4)$ obtains and for all $\mathrm{r}>1 / \mathrm{p}$,

$$
\lim _{n \rightarrow \infty} \int_{R}\left|F_{n}(x)-F_{0}(x)\right|^{r} d x=0 \text {. }
$$

PROOF. Let $r>1 / p$ and set $g(x)=x^{p}, x \geq 0$. Now (2.9) is tantamount to (2.2) and moreover (2.8) holds since pr $>1$. Corollary 2 then follows from Corollary 1 . D

The following example shows that Theorem 1 and Corollary 2 are sharp or best possible results in the sense that (2.5) (resp. (2.10)) can fail if (2.3) is dispensed with (resp. if $r \leq 1 / p$ ).

EXAMPLE. Define distribution functions $F_{0}$ and $F_{n}, n \geq 1$, by

$$
F_{0}(x)=\left\{\begin{array}{ll}
0, & x \leq 0 \\
1, & x>0
\end{array} \text { and } \quad F_{n}(x)=\left\{\begin{array}{cl}
0, & x \leq 0 \\
(n-1) / n, & 0<x \leq n \\
1, & x>n .
\end{array}\right.\right.
$$

Then $\mathrm{F}_{\mathrm{n}} \stackrel{\mathrm{c}}{\rightarrow} \mathrm{F}_{0}$. Set $\phi(\mathrm{x})=\mathrm{x}^{\mathrm{r}}, \mathrm{x} \geq 0$, where $\mathrm{r}>0$. Now if $\mathrm{g}(\mathrm{x})=\mathrm{x}, \mathrm{x} \geq 0$, then (2.2) and (2.9) (with $p=1$ ) obtain since $\int_{R}|x| d F_{n}(x)=1, n \geq 1$. But if $r \leq 1$, then the integral of (2.3) diverges for all $\mathrm{C}>0$ and both (2.5) and (2.10) fail since for $n \geq 1,\left|F_{n}-F_{0}\right|=n^{-1} I_{(0, n]}$ implying

$$
\begin{aligned}
\int_{R} \phi\left(\mid F_{n}(x)\right. & \left.-F_{0}(x) \mid\right) d x=\int_{R}\left|F_{n}(x)=F_{0}(x)\right|^{r} d x \\
& =n^{1-r}+ \begin{cases}\infty, & 0<r<1 \\
1, & r=1 .\end{cases}
\end{aligned}
$$

On the other hand if $r>1$, then (2.3) obtains and

$$
\int_{R} \phi\left(\left|F_{n}(x)=F_{0}(x)\right|\right) d x=\int_{R}\left|F_{n}(x)-F_{0}(x)\right|^{r} d x=n^{1-r}+0
$$

It is well known (see, e.g., Loeve [5, p. 181]) that if $\left\{F_{n}, n \geq 1\right\}$ are distribution functions and $F_{0} \epsilon 3$ then the weak convergence of $F_{n}$ to $F_{0}$ is equivalent to $F_{n}(x)+F_{0}(x)$ for all $x$ in some dense subset of $R$. The following theorem, which will be used to prove a converse to Theorem 1, characterizes weak convergence of distribution functions in terms of their almost everywhere limiting behavior with respect to Lebesgue measure and may be of independent interest.

THEOREM 2. Let $\left\{F_{n}, n \geq 1\right\}$ be distribution functions and let $F_{0} \in \mathfrak{K}$ Then the following are equivalent: 
(i) $\mathrm{F}_{\mathrm{n}} \stackrel{\mathrm{w}}{\rightarrow} \mathrm{F}_{0}$.

(ii) $\mathrm{F}_{\mathrm{n}} \rightarrow \mathrm{F}_{0}$ a.e.

(iii) For every integer subsequence $n(k)+\infty$,

$$
\underset{k \rightarrow \infty}{\lim \inf }\left|F_{n(k)}-F_{0}\right|=0 \text { a.e. }
$$

PROOF. (i) $\Rightarrow$ (ii). This implication is evident since the set of discontinuity points of $\mathrm{F}_{0}$, being countable, is of Lebesgue medsure 0 .

(ii) $\Rightarrow$ (iii). This implication is obvious.

(iii) $\Rightarrow$ (i). Assume that (i) fails. It will be shown that (iii) also fails. Since (i) fails, there exists a point $x_{0}$ in $C\left(F_{0}\right)$, a subsequence $n(k)+\infty$, and a number $\varepsilon>0$ such that either

$$
\mathrm{F}_{\mathrm{n}(\mathrm{k})}\left(\mathrm{x}_{0}\right)-\mathrm{F}_{0}\left(\mathrm{x}_{0}\right) \geq \varepsilon, \text { all } \mathrm{k} \geq 1
$$

or

$$
\mathrm{F}_{\mathrm{n}(\mathrm{k})}\left(\mathrm{x}_{0}\right)-\mathrm{F}_{0}\left(\mathrm{x}_{0}\right) \leq-\varepsilon, \text { all } \mathrm{k} \geq 1
$$

Since $x_{0}$ is in $C\left(F_{0}\right)$, there exists $\delta>0$ such that $\left|F_{0}(x)-F_{0}\left(x_{0}\right)\right| \leq \varepsilon / 2$ for all $x$ in $\left[x_{0}-\delta, x_{0}+\delta\right]$. If $(2.11)$ holds, then for all $x$ in $\left[x_{0}, x_{0}+\delta\right]$ and all $k \geq 1$

$$
\begin{aligned}
\mathrm{F}_{\mathrm{n}(\mathrm{k})}(\mathrm{x})-\mathrm{F}_{0}(\mathrm{x}) & \geq \mathrm{F}_{\mathrm{n}(\mathrm{k})}\left(\mathrm{x}_{0}\right)-\mathrm{F}_{0}\left(\mathrm{x}_{0}\right)-\left(\mathrm{F}_{0}\left(\mathrm{x}_{0}+\delta\right)-\mathrm{F}_{0}\left(\mathrm{x}_{0}\right)\right) \\
& \geq \varepsilon-\varepsilon / 2=\varepsilon / 2
\end{aligned}
$$

impl ying

$$
\left|F_{n(k)}(x)-F_{0}(x)\right| \geq \varepsilon / 2 \text {. }
$$

On the other hand if $(2.12)$ holds, then for all $x$ in $\left[x_{0}-\delta, x_{0}\right]$ and all $k \geq 1$

$$
\begin{aligned}
\mathrm{F}_{\mathrm{n}(\mathrm{k})}(\mathrm{x})-\mathrm{F}_{0}(\mathrm{x}) & \leq \mathrm{F}_{\mathrm{n}(\mathrm{k})}\left(\mathrm{x}_{0}\right)-\mathrm{F}_{0}\left(\mathrm{x}_{0}\right)-\left(\mathrm{F}_{0}\left(\mathrm{x}_{0}-\delta\right)-\mathrm{F}_{0}\left(\mathrm{x}_{0}\right)\right) \\
& \leq-\varepsilon+\varepsilon / 2=-\varepsilon / 2
\end{aligned}
$$

and again (2.13) holds. Thus, if (2.11) prevalls, then (2.13) ensures that

$$
\underset{k \rightarrow \infty}{\lim \operatorname{lnf}}\left|F_{n(k)}-F_{0}\right|>0
$$

on $\left[x_{0}, x_{0}+\delta\right]$ whereas if (2.12) prevails, then (2.13) ensures (2.14) on $\left[x_{0}-\delta, x_{0}\right]$. Since $\left[x_{0}, x_{0}+\delta\right]$ and $\left[x_{0}-\delta, x_{0}\right]$ each have positive Lebesgue measure, (2.14) guarantees the failure of (iii).

Eisenberg and Shixin [6] obtained necessary and sufficient conditions for uniform convergence of distribution functions thereby strengthening a result of Dyson [7] (see, also, Kawata [8, p. 349]) which asserts that if characteristic functions converge uniformly (necessarily to a characteristic function $\psi$ ), then the 
corresponding distribution functions converge unifurmly to the distribution function corresponding to $\psi$.

COROLLARY 3. Let $\left\{F_{n}, n \geq 0\right\}$ be distribution functions and for each $n \geq 0$, let $\mu_{n}$ denote the Lebesgue-Stieltjes measure determined by $F_{n}$ and let $\psi_{n}$ denote the corresponding characteristic function. Then the following are equivalent:

(i) $F_{n}(x) \rightarrow F_{0}(x)$ and $F_{n}(x+) \rightarrow F_{0}(x+)$, all $x \in K$, where $F_{n}(x+)=\lim _{y \nmid x} F_{n}(y), n \geq 0$.

(ii) $\lim _{n \rightarrow \infty} \sup _{x \in R}\left|F_{n}(x)-F_{0}(x)\right|=0$.

(iii) Condition (iii) of Theorem 2 and $\lim _{n \rightarrow \infty} \sum_{x \in R}\left(\mu_{n}(\{x\})-\mu_{0}(\{x\})\right)^{2}=0$.

(iv) Condition (iii) , t Theorem 2 and $\lim _{n \rightarrow \infty} \sup _{x \in R}\left|\mu_{n}(\{x\})-\mu_{0}(\{x\})\right|=0$.

(v) Condition (iii) ,f Theorem 2 and $\underset{n \rightarrow \infty}{\lim } \mu_{n}(\{x\})=\mu_{0}(\{x\})$, all $x \in R$.

(vi) Condition (iii) of Theorem 2 and

$$
\lim _{n \rightarrow \infty} \lim _{T \rightarrow \infty} \frac{1}{2 T} j_{[-T, T]}\left|\psi_{n}(t)-\psi_{0}(t)\right|^{2} d t=0 .
$$

PROOF. (i) $\Rightarrow$ (ii). This implication is proved in Chow and Teicher [3, p.260].

(ii) $\Rightarrow(i i i)$ and $(i i) \Rightarrow(v i)$. These implications follow from Theorem 2 and the tisenberg-Shixin theorem [6].

$$
\begin{aligned}
& \text { (iii) } \Rightarrow(i v) \text { and (iv) } \Rightarrow(v) \text {. These implications are obvious. } \\
& (v i) \Rightarrow(i i) \text {. This implication follows from Theorem 2, the Levy continuity }
\end{aligned}
$$
theorem, and the Eisenberg-Shixin theorem [6].

(v) $\Rightarrow(i)$. This implication follows from Theorem 2 and the fact (proved by Eisenberg [9]) that

$$
\mathrm{F}_{\mathrm{n}} \stackrel{\mathrm{c}}{\rightarrow} \mathrm{F}_{0} \text { and } \lim _{\mathrm{n} \rightarrow \infty} \mu_{\mathrm{n}}(\{\mathrm{x}\})=\mu_{0}(\{\mathrm{x}\}) \text {, all } \mathrm{x} \in \mathrm{R} \Rightarrow \text { (i). }
$$

REMARK. Eisenberg's proof of (2.15) uses Theorem 8.1 .3 of Chow and Teicher [3, p. 255].

The next theorem generalizes Theorem $B$ and is a version of a converse to Theorem 1. Note, however, that there are no assumptions concerning a function $g$ as in Theorem 1.

THEOREM 3. Let $\left\{F_{n}, n \geq 1\right\}$ be distribution functions and let $\phi$ be a nondecreasing function defined on $[0,1]$ with $\phi(0) \geq 0$ and $\phi(x)>0$ for $0<x \leq 1$. If for some $\mathrm{F}_{0} \in \mathbf{3}$

$$
\lim _{n \rightarrow \infty} \int_{R} \phi\left(\left|F_{n}(x)-F_{0}(x)\right|\right) d x=0
$$

then

Moreover,

$$
\mathrm{F}_{\mathrm{n}} \stackrel{\mathrm{c}}{\rightarrow} \mathrm{F}_{0}
$$

$$
\lim _{n \rightarrow \infty} \sup _{x \in R}\left|F_{n}(x)-F_{0}(x)\right|=0
$$


if f

$$
\lim _{n \rightarrow \infty} u_{n}(\{x\})=\mu_{0}(\{x\}), \text { al } 1 \quad x \in K
$$

where $\mu_{n}$ is the Lebesgue-Stifltjes measure determined by $F_{n}, n \geq 0$.

PROOF. Note at the outset that $(2.16)$ and the monotonicity of $\phi$ ensure that necessarily $\phi(0)=0$. It follows immediately from Theoren 2 and Corollary 3 that (2.17) guarantees the equivalence between $(2.18)$ and $(2.19)$. It will be shown' firstly that

$$
\mathrm{F}_{\mathrm{n}} \stackrel{\mathrm{w}}{\rightarrow} \mathrm{F}_{0}
$$

Let $n(k)+\infty$. In view of Theorem 2, it suffices to show that

$$
\underset{k \rightarrow \infty}{\lim \inf }\left|F_{n(k)}-F_{0}\right|=0 \text { a.e. }
$$

Now by Fatou's lemma and (2.16),

$$
\int_{R} \lim _{k \rightarrow \infty} \inf \phi\left(\left|F_{n(k)}(x)-F_{0}(x)\right|\right) d x \leq \lim _{k \rightarrow \infty} \int_{R} \phi\left(\left|F_{n(k)}(x)-F_{0}(x)\right|\right) d x=0
$$

whence

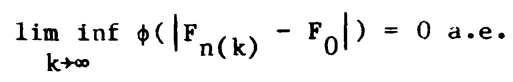

which, taking cognizance of the conditions on $\phi$, implies (2.21) thereby proving $(2.20)$.

It remains to show that $F_{0}$ is a distribution function. Otherwise, either $\lim _{x \rightarrow-\infty} F_{0}(x)>0$ or $\lim _{x \rightarrow \infty} F_{0}(x)<1$. Now if $\lim _{x \rightarrow-\infty} F_{0}(x)>0$, then

$$
\exists \varepsilon>0 \forall n \geq 1 \exists x_{n}<0 \forall x \leq x_{n},\left|F_{n}(x)-F_{0}(x)\right| \geq \varepsilon
$$

and hence $\phi\left(\left|F_{n}(x)-F_{0}(x)\right|\right) \geq \phi(\varepsilon)>0$ implying $\int_{R} \phi\left(\left|F_{n}(x)-F_{0}(x)\right|\right) d x=\infty$ and contradicting $(2.16)$. The case $\lim _{x \rightarrow \infty} F_{0}(x)<1$ is handled similarly.

REMARK. It is well known (see, e.g., Chow and Teicher [3, p. 260]) that (2.17) and $F_{0}$ continuous imply (2.18). Alternatively, it is easy to directly verify that $(2.17)$ and $F_{0}$ continuous imply (2.19).

3. GLOBAL VERSIONS OF THE CENTRAL LIMIT THEOREM.

Throughout this section, let $\left\{x_{n}, n \geq 1\right\}$ be independent random variables defined on a probability space $(\Omega, \mathcal{A}, \mathrm{P})$ and suppose that $\operatorname{EX}_{n}=0,0<\operatorname{EX}_{n}^{2}<\infty, n \geq 1$. Write $s_{n}=\Sigma_{j=1}^{n} X_{j}, s_{n}^{2}=\Sigma_{j=1}^{n} E X_{j}^{2}, n \geq 1$, and let $F_{n}$ denote the distribution function of $s_{n} / s_{n}, n \geq 1$, and $\Phi$ denote that of the $N(0,1)$ distribution. Now it is well known that if $\left\{X_{n}, n \geq 1\right\}$ are also identically distributed, then they obey the CLT

that is,

$$
s_{n} / s_{n} \stackrel{d}{\rightarrow} N(0,1)
$$

$$
\mathrm{F}_{\mathrm{n}} \stackrel{\mathrm{c}}{\rightarrow} \Phi
$$

which is indeed tantamount to

$$
\lim _{n \rightarrow \infty} \sup _{x \in R}\left|F_{n}(x)-\$(x)\right|=0
$$


since $\Phi$ is continuous. By applying Theorem A, Agnew [1] shows under the same assumptions of i.i.d. $\left\{x_{n}, n \geq 1\right\}$ that

$$
\lim _{n \rightarrow \infty} \int_{R}\left|F_{n}(x)-\Phi(x)\right|^{r} d x=0
$$

for all $r>1 / 2$. Agnew refers to this as a global version of the CLT.

This result was generalized by Esseen [10] to the case where the random variables $\left\{x_{n}, n \geq 1\right\}$ can have different distributions provided that they obey the CLT (3.1). Specifically, Esseen proved that if (3.1) prevails, then (3.3) holds for all $r>1 / 2$. It may be noted that Agnew's global version of the CLT and Esseen's generalization of it can also be obtained directly from Corollary 2 (with $p=2$ ) since

$$
\sup _{n \geq 1} \int_{R} x^{2} d F_{n}(x)=\sup _{n \geq 1} L\left(\frac{s_{n}}{s_{n}}\right)^{2}=1<\infty .
$$

Moreover, if $\left\{x_{n}, n \geq 1\right\}$ obeys the classical Lindeberg condition (which is stronger than (3.1)), then, for all $\mathbf{r}>1 / 2,(3.3)$ follows readily from Lemma 4 of Embrechts and Maejima [11] which gives a nonuniform bound on the error in the CLT.

In Theorem 4 below, conditions are given which ensure (3.3) for particular values of $r$ in $(0,1 / 2]$. Of course, the smaller the value of $r>0$, the stronger is (3.3). The proof of Theorem 4 utilizes a famous theorem of Bernstein [12] asserting that if $\left\{x_{n}, n \geq l\right\}$ obeys the Liapounov condition

$$
\sum_{j=1}^{n} E\left|X_{j}\right|^{P}=o\left(s_{n}^{p}\right)
$$

for some $p>2$, then

$$
\lim _{n \rightarrow \infty} E\left|\frac{S_{n}}{s_{n}}\right|^{p}=\int_{R}|x|^{p} d \Phi(x)<\infty .
$$

An alternative proof of Bernstein's theorem, using characteristic functions, was discovered by Brown [13].

When $2<\mathrm{p} \leq 3$, Theorem 4 can be obtained from an inequality of Bikyalis [14] which also appears in Petrov [15, p. 132]. Theorem 4 was proved by Bhattacharya [16] in the case $\mathrm{p} \geq 3$ and $\operatorname{EX}_{\mathrm{n}}^{2}=1, \mathrm{n} \geq 1$. Moreover, Theorem 4 can also be proved using Bernstein's theorem and an inequality of Kolodyaźnyl [17]. This inequality may also be found in Petrov [15, Theorem 9, p. 121].

THEOREM 4. If $\left\{x_{n}, n \geq 1\right\}$ obeys the Liapounov condition (3.4) for some $p>2$, then the global version (3.3) of the CLT obtains for all $r>1 / p$.

PROOF. It is wel1 known (see, e.g., Chow and Teicher [3, p. 293]) that (3.4) with $p>2$ ensures (3.2). Now (3.5) obtains by Bernstein's theorem and hence

$$
\sup _{n \geq 1} \int_{R}|x|^{P} \mathrm{dF}_{n}(x)=\sup _{n \geq 1} E\left|\frac{S_{n}}{s_{n}}\right|^{p}<\infty .
$$

The conclusion then follows immediately from Corollary 2.

The next corollary has, in essence, been obtained by de Acosta and Giné [18]. Furthermore, if $E\left|x_{1}\right|^{P}<\infty$ for some $p \geq 3$, the corollary follows readily from an inequality of Osipov [19] which also appears in Petrov [15, Theorem 13, p. 125]. 
COROLLARY 4. Let $\left\{X_{n}, n \geq 1\right\}$ be i.i.d. random variables with $\operatorname{EX}_{1}=0$, $0<E\left|X_{1}\right|^{P}<\infty$ for some $p \geq 2$ and let $F_{n}$ denote the distribution function of $\sum_{j=1}^{n} x_{j} /\left(n E x_{1}^{2}\right)^{1 / 2}, n \geq 1$. Then (3.3) obtains for all $r>1 / p$. Moreover, if $E\left|X_{1}\right| P\langle\infty$ for all $P>0$, then (3.3) obtains for all $r>0$.

PROOF. To prove the first assertion, let $r>1 / p$. If $p=2$, then (3.3) holds by the Agnew global CLT [1] or by Corollary 2 as already discussed, whence it will be assumed that $p>2$. Since condition (3.4) is then automatic, (3.3) follows directly from Theorem 4. To prove the last assertion, let $r>0$, choose $p \geq 2$ large enough so that $r>1 / p$, and simply apply the portion of the corollary already proved.

ACKNOWLEDGEMENTS. The author expresses great appreciation to Professor Bennett Eisenberg of Lehigh University for informing him that (2.15) is true and in fact proving it. The author also thanks Professor Walter Smith of the University of North Carolina for some helpful comments on an earlier draft of this paper.

\section{REFERENCES}

1. AGNEW, R.P. Global Versions of the Central Limit Theorem, Proc. Nat. Acad. Sci. U.S.A. 40 (1954), 800-804.

2. KRUGLOV, V.M. Convergence of Numeric Characteristics of Sums of Independent Random Variables and Global Theorems, In Proceedings of the Second JapanUSSR Symposium on Probability Theory (Kyoto, Japan, August 2-9, 1972; Ed. G. Maruyama and Yu. V. Prokhorov), Lecture Notes in Math. 330, 255-286, Springer-Verlag, Berlin, 1973.

3. CHOW, Y.S. and TEICHER, H. Probability Theory: Independence, Interchangeability, Martingales, Springer-Verlag, New York, 1978.

4. NISHIMURA S. An Inequality for a Metric in a Random Collision Process. J. Appl. Probab. 12 (1975), 239-247.

5. LOĖVE, M. Probability Theory, Vol. I, 4th ed., Springer-Verlag, New York, 1977.

6. EISENBERG, B. and SHIXIN, G. Uniform Convergence of Distribution Functions, Proc. Amer. Math. Soc. 88 (1983), 145-146.

7. DYSON, F.J. Fourier Transformations of Distribution Functions, Canad. J. Math. $\underline{5}$ (1953), 554-558.

8. KAWATA, T. Fourier Analysis in Probability Theory, Academic Press, New York, 1972.

9. EISENBERG, B. Private communication (1985).

10. ESSEEN, C.G. On Mean Central Limit Theorems, Kungl. Tekn. Högsk. Handl. Stockholm (Trans. Roy. Inst. Tech. Stockholm) No. 121 (1958), 1-31.

11. EMBRECHTS, P. and MAEJIMA, M. The Central Limit Theorem for Summability Methods of I.I.D. Random Variables, Z. Wahrsch. verw. Gebiete 68 (1984), 191-204.

12. BERNSTEIN, S.N. Quelques Remarques Sur le Theoreme Limite Liapounoff, C.R. (Doklady) Acad. Sci. URSS (N.S.) 24 (1939), 3-8.

13. BROWN, B.M. Characteristic Functions, Moments, and the Central Limit Theorem, Ann. Math. Statist. 41 (1970), 658-664.

14. BIKYALIS, A. Estimates of the Remainder Term in the Central Limit Theorem, Litovsk. Mat. Sb. 6 (1966), 323-346 (in Russian).

15. PETRoV, V.V. Sums of Independent Random Variables, Springer-Verlag, New York, 1975.

16. BHATTACHARYA, R.N. On Errors of Normal Approximation, Ann.Probab. 3 (1975), 815-828.

17. KOLODYAŽNYİ, S.F. A Generalization of a Theorem of Esseen, Vestnik Leningrad. Univ. 23, No. 13, (1968), 28-33. (English translation in Vestnik Leningrad Univ., Math. 1, No. 3, (1974), 189-195).

18. DE ACOSTA, A. and GINE, E. Convergence of Moments and Related Functionals in the General Central Limit Theorem in Banach Spaces, Z. Wahrsch. verw. Gebiete 48 (1979), 213-231.

19. OSIPOV, L.V. Asymptotic Expansions in the Central Limit Theorem. Vestnik Leningrad. Univ. 22, No. 19, (1967), 45-62 (in Russian). 


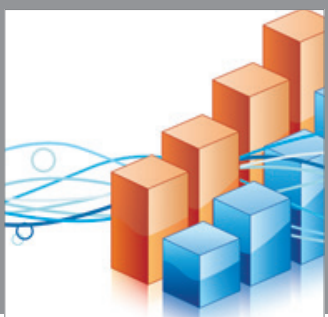

Advances in

Operations Research

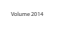

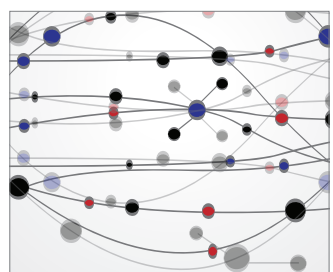

\section{The Scientific} World Journal
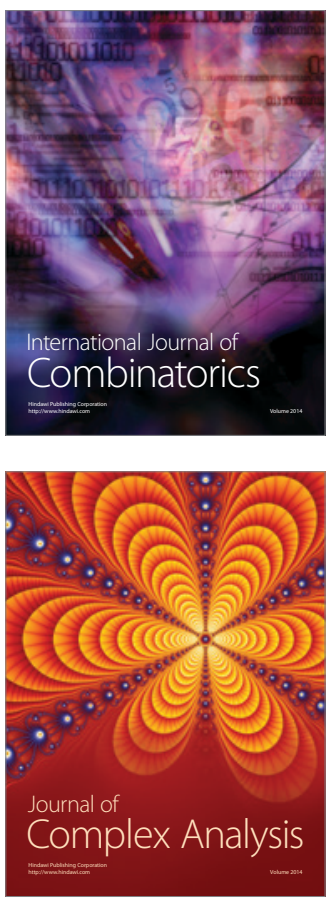

International Journal of

Mathematics and

Mathematical

Sciences
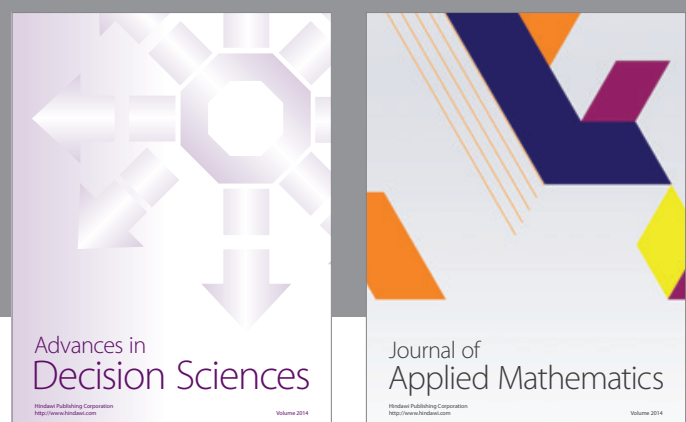

Journal of

Applied Mathematics
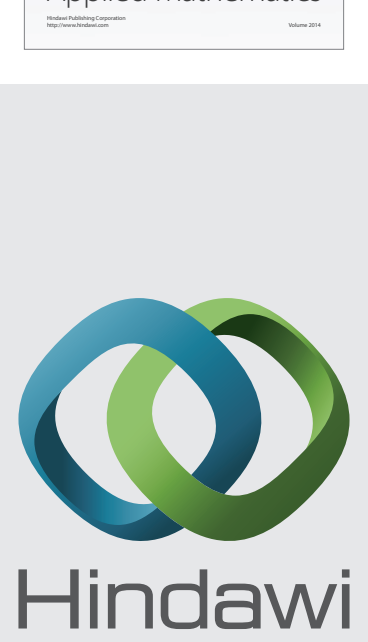

Submit your manuscripts at http://www.hindawi.com
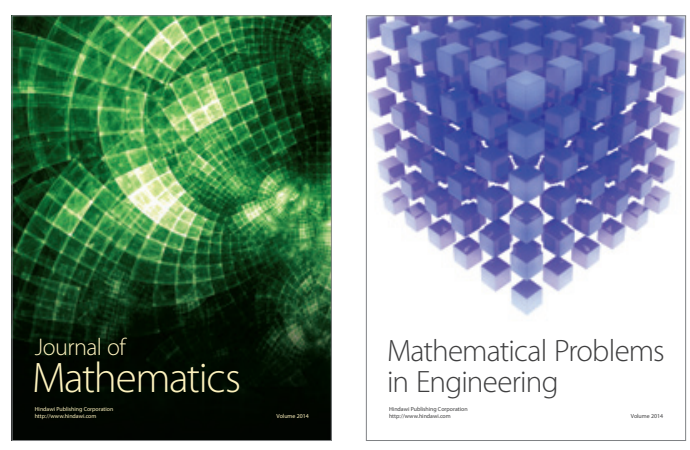

Mathematical Problems in Engineering
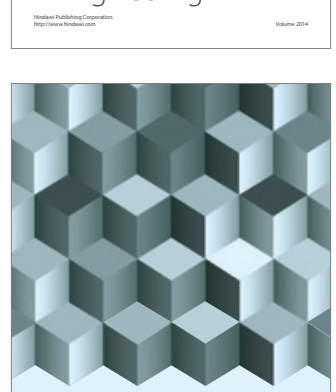

Journal of

Function Spaces
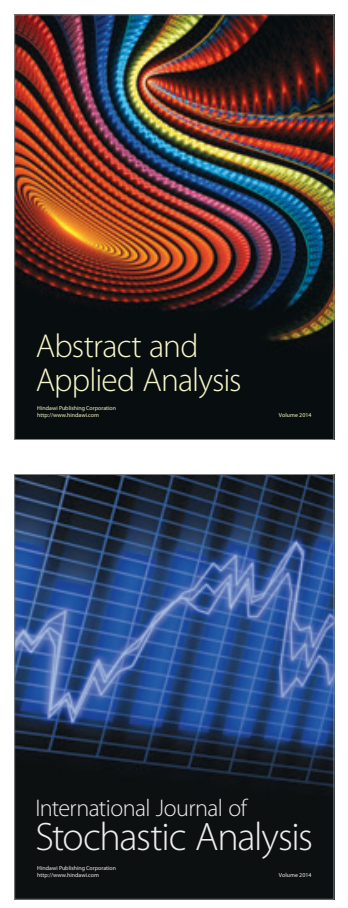

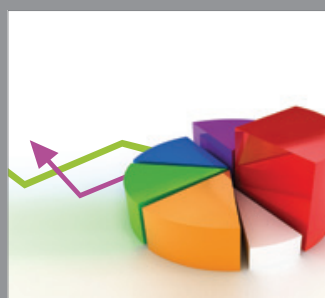

ournal of

Probability and Statistics

Promensencen
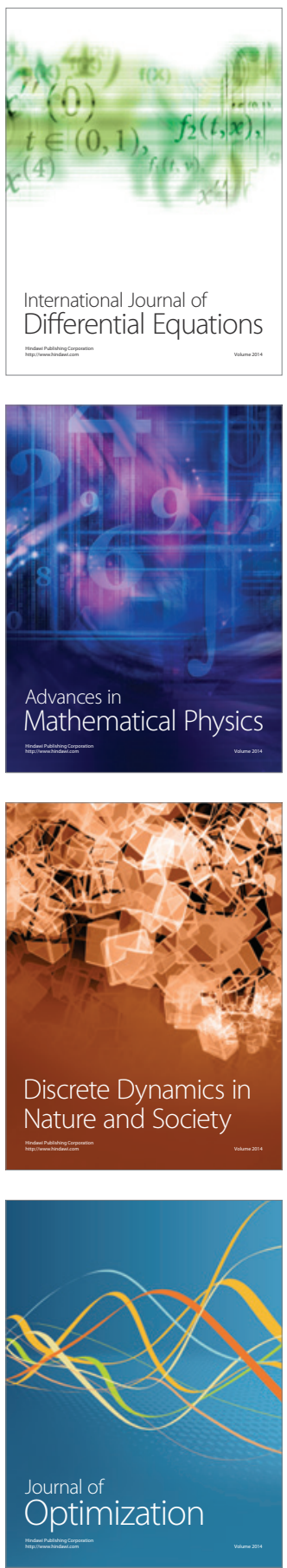